\title{
Philosophie, Politik und wissenschaftliche Weltauffassung:Zur Frage der Philosophie in Österreich und Deutschland
}

\author{
Barry Smith
}

from: Grazer Philosophische Studien 58/59 (2000), 241-262

\section{Einfuihrung}

Eine der merkwürdigsten philosophischen Erscheinungen der letzten zwanzig Jahren ist die sogenannte 'Continental Philosophy' (C.P.), eine Schöpfung vor allem der nordamerikanischen Universitätsszene unserer Zeit. Vorlesungen unter dem Titel 'Continental Philosophy' werden im angelsächsischen Raum alljährlich vielen Tausenden von Philosophiestudenten angeboten, eine Praxis, die zumindest insofern fragwürdig erscheint, als diese Vorlesungen nicht von der Philosophie in Kontinentaleuropa in ihrer Gesamtheit handeln, sondern nur von einem engen Ausschnitt französisch-deutscher Philosophie, wobei Heidegger oft als der einzige feste Punkt erscheint. Um ihn herum wird eine Serie Pariser Denker versammelt, von denen jede Generation den Anspruch erhebt, das 'Ende' der Philosophie (oder der 'Moderne', des 'Autors', des 'Menschen', der 'Identität' u. dgl.) herbeigeführt zu haben. ${ }^{1}$ Bei all dem wird der späte Husserl manchmal als C.P.-Vorläufer berücksichtigt, sein Lehrer Franz Brentano aber ebensowenig wie andere prominente deutsche Philosophen des 20. Jahrhunderts wie etwa Ernst Cassirer oder Nicolai Hartmann. Französische Philosophen in der Tradition von Poincaré, Bergson oder Gilson werden gleichfalls, nicht anders als polnische, skandinavische oder tschechische Philosophen, ignoriert.

Was fungiert als Einheitsmoment dieser 'Continental Philosophy'? Was haben Heidegger, Derrida und Luce Irigaray gemeinsam, das sie von Phänomenologen wie etwa Reinach oder Ingarden oder dem sagenhaften Johannes Daubert ${ }^{2}$ unterscheidet? Die naheliegende Antwort ist: Antipathie gegenüber der Wissenschaft oder besser: Antipathie gegenüber den gewöhnlichen Normen der Evidenz und des Argumentierens sowie gegenüber den üblichen bürgerlichen Zwecken der Universität, dazu die Ersetzung - wenigstens im Fall vieler französischen Philosophen, die als ‘Kontinentalphilosophen’ anerkannt werden - von Wissenschaft in diesem breiteren Sinn durch Politik.

\footnotetext{
${ }^{1}$ Vgl. Engel 1994.

${ }^{2}$ Schuhmann und Smith 1985, Schuhmann 1998.
} 


\section{Der Fall Österreich}

Betrachten wir nun das Schicksal der Philosophie in Österreich. Das erste, was uns dabei auffällt, ist, daß es Philosophen aus Österreich bei der Zulassung zum Pantheon der 'Kontinentalphilosophen' besonders schlimm erging. Warum ist dem so? Warum, um die Frage von einer anderen Seite her zu stellen, gibt es in (Alt-)Österreich eine so enge Verbindung zwischen Philosophie und Wissenschaft? Bernard Bolzano, Ernst Mach, Ludwig Boltzmann, Ludwig Wittgenstein, der Wiener Kreis, Karl Popper, Michael Polanyi, Paul Feyerabend, Wolfgang Stegmüller, Rudolf Haller - das ist doch eine beeindruckende Liste, und zwar auch dann, wenn man mit den Ansichten gewisser Philosophen in dieser Liste nicht in jeder Beziehung einverstanden ist.

Bevor wir eine Antwort auf diese Frage zu formulieren versuchen, muß daran erinnert werden, daß auch in Österreich, und auch im tiefsten Dickicht des Wiener Kreises, die rein wissenschaftliche Orientierung nicht immer ohne politischen Zusatz blieb. Als 1929 das Manifest des Wiener Kreises unter dem Titel "Wissenschaftliche Weltauffassung" veröffentlicht wurde, war Schlick, dem diese Schrift gewidmet war, gerade deswegen nicht zufrieden, weil er jegliche Auffassung des Kreises als 'Bewegung' unsympathisch fand und eine bescheidenere, streng wissenschaftliche Art des Herangehens an die Probleme bevorzugte. Wie Heinrich Neider es 1977 in einem Interview mit Haller und Rutte formulierte: 'Schlick haßte alles, was nach Agitation aussah, war gegen jede Agitation.'

Wir haben es nicht notwendig, zu agitieren, das überlassen wir politischen Parteien, in der Wissenschaft sagen wir, was wir gefunden haben, wir hoffen die Wahrheit zu sagen: wenn es die Wahrheit ist, wird sie siegen. (Haller und Rutte 1977, S. 31)

Neurath dagegen propagierte die politische Agitation. Er war jemand, der

looked at everything - ideas as well as facts - through an often distorting lens of socialist philosophy and with an eye to the possible effects of the ideas and facts on a socialization of society. I have never seen a scholar as consistently obsessed with an idea and an ideal as Neurath. (Menger 1994, p. 60)

'Für die proletarische Front deckt sich,' wie das ehemalige Mitglied des Zentralplanungsamts der zeitweiligen Bayerischen Sowjetrepublik in München verkündet, 'Kampftechnik und Propagandainteresse mit Hochhaltung der Wissenschaft und Überwindung der Metaphysik' ${ }^{3}$

\section{Wahrheit contra Agitation}

Es gibt also innerhalb der real existierenden wissenschaftlichen Philosophie in Österreich eine Spannung zwischen Wissenschaft auf der einen Seite und Politik auf der anderen. Diese Spannung ist allerdings subtil, denn das, zu dessen Gunsten man hier agitiert, ist gerade die Wahrheit und die Wissenschaft. Wo Schlick auf

\footnotetext{
${ }^{3}$ Neurath 1981, Bd. I, S. 355, auch zitiert durch Haller 1993, S. 157, Kursivierung im Original.
} 
einer streng wissenschaftlichen Auffassung der Philosophie des Wiener Kreises beharrte, war für Neurath der Wiener Positivismus in erster Linie in politischem Licht zu betrachten. Friedrich Stadler vertritt in diesem Zusammenhang die Auffassung, daß das geistige Wien in der Zeit zwischen den Kriegen in 'zwei Lager' gespalten war:

während einerseits imBereich wissenschaftlicher Philosophie demokratische (aufklärerische, liberale, sozialistische) Tendenzendominieren, umspannt das Spektrum auf der anderen Seite fast alle Formen antidemokratischer Gesinnung, vom neoromantischen Konservatismus bis zu faschistisch-totalitären Auswüchsen. Somit liegt es nahe, das philosophische Leben in den heftigen parteipolitischen Kulturkampf zwischen dem bürgerlichen Lager und der Arbeiterbewegung jener Zeit einzuordnen (Stadler 1979, S. 42). ${ }^{4}$

Eine ähnliche These vertritt auch Alfred Ayer, der den Wiener Kreis im Jahre 1932 während seiner Flitterwochen in Österreich kennenlernte:

The members of the Vienna Circle, with the notable exception of Otto Neurath, were not greatly interested in politics, but theirs was also a political movement. The war of ideas which they were waging against the Catholic church had its part in the perennial Viennese conflict between the socialists and the clerical reaction. (Ayer 1977, S. 129)

Und wie Johann Dvorak es formulierte:

Angesichts der Tatsache, daß das Bürgertum - insbesondere in Mitteleuropa - sich aller aufklärerischen Traditionen entledigt hatte und den Kulten des Irrationalismus huldigte, während das Proletariat eine rationale Gestaltung der Gesellschaft anstrebte, bestand durchaus die Hoffnung, [daß] “Gerade das Proletariat ... zum Träger der Wissenschaft ohne Metaphysik” [würde]. (Dvorak 1985,

S. 142, Zitat aus Neurath1981, S. 293)

In bezug auf die allgemeine Verfassung der österreichischen Gesellschaft zwischen den Kriegen mag eine solche 'Zweilager'-These eine gewisse Plausibilität haben, zumalnicht nur 'Sozialismus' und 'Kommunismus', sondern auch 'Liberalismus', 'Rationalismus' und sogar 'Logizismus' im damaligen Feindbild des rechten Lagers einbegriffen waren. Unhaltbar ist allerdings die Neurathsche Unterstellung, daß das Aufblühen wissenschaftlicher Philosophie im Wien dieser Zeit als Ausdruck des österreichischen Sozialismus oder Antiklerikalismus oder als Teil 'einer nicht-kapitalistischen Vergesellschaftung von Wissenschaft, einer Demokratisierung von Wissenschaft' ${ }^{5}$ zu erklären sei. Schließlich nahmen ähnliche sozialistische und antiklerikale Bewegungen beispielsweise in Spanien oder Italien keine vergleichbaren Formen an. Eine zu

\footnotetext{
${ }^{4}$ Man beachte den bedauernswerten Umschlag zwischen dem ersten, sorgfältig formulierten Satz dieser Passage und der zweiten, ideologisierten Vereinfachung der Sachlage. Stadler 1995 enthält eine überlegtere Darstellung, die den breiteren, nicht ausschließlich sozialistischen Zusammenhang des österreichischen Positivismus der Zwischenkriegszeit berücksichtigt.

${ }^{5}$ Dvorak 1985, S. 133f. Vgl. auch S. 139f., wo Dvorak die Ableitung der Idee der Einheitswissenschaft aus dem historischen Materialismus unternimmt.
} 
enge Konzentrierung auf die 'Zweilager'-Idee führt, wie nicht zuletzt die einschlägige Literatur des Instituts Wiener Kreis selber bezeugt, zudem zu einer Vernachlässigung der Rolle der Brentanisten und anderer vom Sozialismus entfernter Denker bei der Vorbereitung einer wissenschaftsfreundlichen Universitätslandschaft nicht nur in Wien, sondern auch in anderen mitteleuropäischen Universitäten.

Der Hauptmangel der Behauptung, der Wiener Positivismus sei das Produkt einer sozialistischen 'Vergesellschaftung von Wissenschaft', besteht allerdings darin, daß sie der Tatsache nicht gerecht zu werden vermag, daß nur ausgesprochen wenige wichtige wissenschaftliche Philosophen in Österreich von sozialistischer Gesinnung waren. ${ }^{6}$

Was die Philosophen in Wien zwischen den Kriegen anbelangt, müßen wir eigentlich drei Gruppen unterscheiden: die Linken (Neurath), die Rechten ('christliche Sozialisten', Othmar Spann, et al., die an der Universität vor allem in den medizinischen und juristischen Fakultäten dominant waren), und jene, die dazwischenlagenund einenLiberalismus englischerPrägung vertraten (Schlick, Mises, Hayek, Popper). ${ }^{7}$ Wie die Geschichte gezeigt hat, war die Stellung dieser dritten Gruppe unter den damals gegebenen Umständen eher schwach. Auf lange Sicht hat sich jedoch ihre überragende Bedeutung herausgestellt.

Es ist zwar interessant, daß Neuraths Befürwortung einer Abschaffung der Geldwirtschaft zugunsten einer 'Naturalwirtschaft' Friedrich Hayek davon abhielt, Annäherungsversuche beim Schlick-Kreis zu machen, auch nachdem sein Interesse durch seinen Freund und Klubkameraden beim Ludwig von Mises-Kreis, Felix Kaufmann, erweckt worden war. Interessant ist weiterhin die Tatsache, daß die Wiener wissenschaftliche Philosophie (vor allem in Form des Denkens vonErnst Mach) einen gewissenEinfluß auf Austro-Marxisten wie Friedrich Adler ausüben konnte. Auch Otto Bauer hat die Arbeit des Wiener Kreises in dem Maße geschätzt, daß er den logischen Positivismus als Wegweiser sogar für den marxistischen Materialismus betrachtete. Die Idee einer Zwei-Lager-Theorie, die alle ehrlichen, wissenschaftlich orientierten Denker in Wien, zusammen mit allen fortschrittlichen Strömungen wie Positivismus, Naturwissenschaft und der Wiener sozialistischen Stadtregierung, in eine Linie bringen will und sie vereint dem Katholizismus, AustroFaschismus, der bäuerlichenDummheit und anderen dunklen Mächten gegenüberstellt, bricht jedoch gerade durch die Konfrontation mit liberalen oder konservativen Intellektuellen wie Schlick, Kraft, Waismann, Menger, Kaufmann und auch Wittgenstein zusammen.

\section{4. $\quad$ Ein spätes Aufblühen des Liberalismus}

\footnotetext{
${ }^{6}$ Außer Neurath und seinem Schwager Hahn, dem "Vorsitzenden des Bundes der sozialistischen Professoren", waren Carnap, Frank und Zilsel Sozialisten. Sozialisten waren auch im Institut Karl Bühlers vertreten, z.B. durch Lazarsfeld und Jahoda.

${ }^{7}$ Wie Neider es formulierte:

Schlick war überhaupt ein Mann, der zu Politik und Staat kein Verhältnis hatte, er war ein Liberaler im alten Sinn, für den höchstens Feuerwehr und Polizei als notwendige Übel zugelassen waren. Aber ansonsten braucht man den Staat nicht. (Haller and Rutte, S. 24)
} 
Wie sollen wir also die Dominanz einer wissenschaftlich orientierten Philosophie in Österreich, und vor allem im Wien der Zwischenkriegszeit, erklären? Eine Antwort auf diese Frage, die durch J. C. Nyíri vertreten wurde, lautet: Österreich hinkte um die Jahrhundertwende in Sachen Wissenschaft hinter seinen entwickelteren westlichen Nachbarn her. Das Kaiserreich erlebte einen verhältnismäßig späten Prozeß der Urbanisierung, was auch eine verspätete Entwicklung jener liberalen Gewohnheiten und Werte mit sich brachte, die die Voraussetzung einer modernen wissenschaftlichen Weltanschauung sind. Es fehlte also in Österreich auch an solchen Forschungseinrichtungen, wie es sie in Deutschland schon seit geraumer Zeit gab. Auf der anderen Seite machte sich mit der Etablierung einer liberalen Einstellung in Österreich auch das Verlangen nach den Ausschmückungen einer modernen aufgeklärten Kultur bemerkbar. Österreichwar aber nicht in der Lage, die Mittel für die Schaffung bedeutsamer Institutionen der Wissenschaft im engeren Sinn aufzubringen, und dies ergab, wie Nyíri es formulierte, 'a vacuum which the theory of a practice so attractively pursued elsewhere could then fill' (1986, S. 143).

Die Nyíri-These läßt sich besonders klar durch den Fall Boltzmanns veranschaulichen, dem die Mittelknappheit für experimentelle Arbeit anscheinend dazu zwang, sich viel stärker den billigeren Gebieten der theoretischen Physik und Philosophie zuzuwenden. Diese These hat jedoch ihre Probleme. Erstens kann schon die Behauptung, daß der Modernisierungsprozess in Österreich später als in Deutschland verlief, in Frage gestellt werden. ${ }^{8}$ Weiter vollzog die liberale und wissenschaftliche Aufklärung sich in England, Frankreich und Holland doch vor der massiven Verstädterung, die ja selbst in großem Maße ein Produkt von Wissenschaft und Liberalismus war. Vor allemaber muß man konstatieren, daß es überhaupt problematisch ist, die Erklärung von intellektuellen oder kulturellen Entwicklungen primär durch Bezugnahme auf zugrundeliegende soziale oder ökonomische Faktoren zu versuchen. Erklärungen dieser Art führten einige marxistische und andere Denker in die Irre, die an eine im weiteren Sinne ökonomische Auffassung des menschlichen Handelns geglaubt hatten. Wo wir es aber mit komplexen Denkbewegungen zu tun haben, können solche Erklärungen bestenfalls nur partiell sein. Sie werden uns nur selten den erforderlichen Einblick in den genauen intellektuellen Inhalt der fraglichen Bewegungen gewähren. Warum mußte etwa der in Österreich bevorzugte Ersatz für genuin wissenschaftlichen Fortschritt genau diese (phänomenalistischen und physikalistischen) Formen annehmen? Wodurch soll die eigenartige Mischung aus britischemEmpirismus und Russellscher Logik erklärt werden, die jene grundlegende Struktur lieferte, in deren Rahmen die verschiedenen Mitglieder des Schlick-Kreises ihre Ideen entwickelten?

Offensichtlich müssen wir trotz der Dominanz von Schulen, Bewegungen und Institutionen auf die Rolle von Einzelpersonen hinweisen, wenn wir zufriedenstellende Antworten auf Fragen dieser Art geben wollen. Und es gibt zweifellos eine beträchtliche Anzahl solcher Persönlichkeiten, die einem in diesemZusammenhang

\footnotetext{
${ }^{8}$ Vgl. etwa Good 1984.
} 
sofort einfallen. Es seinochmals an Boltzmann, dessen Vision einer einheitlichenUniversalwissenschaft großen Einfluß auf die geistige Welt Wiens ausübte, sowie an Wittgenstein erinnert, dessen Tractatus einen nicht unbeträchtlichen Einfluß sowohl auf Schlick als auch auf Carnap ausübte, und zwar genau in den wichtigen Gründerjahren des Wiener Kreises. Wir können wohl davon ausgehen, daß es keine erschöpfende soziale oder ökonomische Erklärung der herausragenden Kreativität eines Boltzmann oder Wittgenstein (oder Gödel oder Einstein) geben kann. Auch können wir davon ausgehen, daß es keine solche Erklärung der eigentümlichen Langlebigkeit Brentanos (1838-1917) und der wichtigstenMitglieder seines weiteren Kreises geben kann, Denker - wie Marty (1847-1914), Stumpf (1848-1936), Meinong (1853-1920), Höfler (1853-1922), Husserl(1859-1938), Ehrenfels (1859-1932) und Twardowski (1866-1938) -, die so viel für die Verbreitung der Botschaft der wissenschaftlichen Philosophie im Kaiserreich und darüber hinaus geleistet haben. ${ }^{9}$

\section{Die Neurath-These}

Auch wenn all das zugegeben wird, wird es aber umgekehrt nicht genügen, Individuen in völliger Isolation von ihrembreiteren gesellschaftlichen und institutionellenZusammenhang zu betrachten. Nicht nur deswegen, weil das Individuum durch die umliegende Kultur mitgeformt wird, sondernauch, weil seine Ideen erst in dem Maß Wurzeln schlagen, in dem sie im Denken ihrer Adressaten auf Verständnis stoßen. Noch wichtiger ist allerdings die Tatsache, daß eine Einzelperson, wie genial und folgenreich sie auch immer sein mag, die Fähigkeit, ihre Zeitgenossen zu beeinflussen, nur insofern besitzt, als es Institutionen gibt, die die Verbreitung ihrer Ideen ermöglichen.

Es besteht also das Bedürfnis nach einer gemischten Erklärung, die sowohl institutionellen und sozialpolitischen Faktoren als auch der zufälligen Rolle von Individuen einen Platz einräumt. Grundelemente einerüberzeugenden Erklärung dieser Art hat Neurath (entgegen dem, was er selbst in seinen eher politischen Pamphleten vorgebracht hat) in dem Abschnitt "Vorgeschichte" des schon erwähnten Manifests des Wiener Kreises geliefert.

Wien, argumentieren Neurath und seine Mitautoren Carnap und Hahn, war ein besonders fruchtbarer Boden für den Aufstieg der wissenschaftlichen Philosophie, weil der bürgerliche Liberalismus der zweiten Hälfte des 19. Jahrhunderts in Österreich eine antimetaphysische Haltung verkörperte, die aus dem Geist der Aufklärung, des Utilitarismus und der Freihandelsbewegung Englands erwachsen war. Auch Mach war ein Produkt dieser liberalen Aufklärung, und dieselbe antimetaphysische Haltung zeigt sich auch in seinen Versuchen, alle metaphysischen Begriffe aus den empirischen Wissenschaften zu verbannen. Der Einfluß Machs und seines Nachfolgers Boltzmann, argumentiert Neurathweiter, macht es verständlich, warum gerade

\footnotetext{
${ }^{9}$ Vgl. hierzu Kapital 1 meines 1994.
} 
in Wien ein lebendiges Interesse an epistemologischen und logischen Problemen im Bereich der Grundlagen der Physik bestand. Hayek z.B. berichtet, daß er und seine Zeitgenossen in der Zeit kurz nach dem Krieg in Wien 'in Mach fast die einzigen Argumente gegen eine metaphysische und nebelhafte Einstellung' fanden, die das Denken der in dieser Zeit dominanten Universitätsphilosophen wie Othmar Spann charakterisierte.

Der Weg von Mach ging dann eher zu Helmholtz, zu Poincaré und ähnlichen Denkern, und für die, die es systematisch betrieben, wie mein Freund Karl Popper, natürlich zu den ganzen Naturwissenschaftlern und Philosophen dieser Zeit (Hayek 1966, S. 42f.).

Neurath erwähnt weiter einige Wiener Sozialphilosophen sowohl des marxistischen als auchdes bürgerlichen Lagers, die im späten 19. Jahrhundert 'bewußt im Geist der Aufklärung' gewirkt hatten. Auch im Bereich der Nationalökonomie wurde, wie Neurath konstatiert, eine streng wissenschaftliche Methode durch die Schule der Grenznutzentheorie eingeführt, die 1871 durch Carl Menger (Vater von Karl) an der Wiener Universität gegründet worden war.

Neurath erwähnt in seiner Darstellung aber auch die Rolle Franz Brentanos. Wie er selbst formuliert, war der Boden für die Bemühungen des Wiener Kreises in Richtung einer Reform der Logik und einer Beschäftigung mit Grundlagenproblemen auch durch Brentano vorbereitet worden:

Brentano hatte als katholischer Geistlicher Verständnis für die Scholastik; er knüpfte unmittelbar an die scholastische Logik und an die Leibnizschen Bemühungenumeine Reformder Logik an, während er Kant und die idealistischenSystemphilosophen beiseite ließ. Das Verständnis Brentanos und seiner Schüler für Männer wie Bolzano (Wissenschaftslehre, 1837) und andere, die sich um eine strenge Neubegründung der Logik bemühten, ist immer wieder deutlich zutage getreten. (Neurath, Carnap und Hahn 1929, S. 302)

Auch Brentano ist der liberalen Bewegung des 19. Jahrhunderts zuzurechnen. Er war z.B. daran beteiligt, daß der junge Sigmund Freud mit der Übersetzung eines der Bände der Gomperz-Edition der Werke J. St. Mills (über Platon, Sozialismus und Frauenemanzipation) beauftragt wurde. (Freud war eine Zeitlang treuer Anhänger der Philosophie Brentanos. ${ }^{10}$ ) Eine merkwürdige Unterstützung der Auffassung Neuraths von der Bedeutung Brentanos liefert in diesemZusammenhang die Tatsache, daß die Zentren der wissenschaftlichen Philosophie in Europa - Wien, Prag, Lemberg, Graz, Berlin, Göttingen - genau die Städte waren, in denen die ausgezeichnetsten Studenten Brentanos seit den neunziger Jahren des 19. Jahrhunderts Lehrstiuhle innehatten.

\section{Die Neurath-Haller-These}

\footnotetext{
${ }^{10}$ Vgl. Hemecker 1991; zu einem möglichen philosophischen Einfluß Brentanos auf Freuds Psychoanalyse vgl. auch Flores-Morelos 1996.
} 
Nicht nur verteidigte Brentano die streng wissenschaftliche Methode in der Philosophie, er teilte mit den britischen Empiristen und den Wiener Positivisten auch eine gewisse antimetaphysische Einstellung und zeigte eine besonders starke Antipathie gegen die 'mystische Afterphilosophie' der deutschen Idealisten. Seine Arbeit verwendet zudem Methoden der Sprachanalyse, die in gewisser Hinsicht denenähneln, die später in England entwickelt wurden.

Rudolf Haller hat nundiese Neurathsche Darstellung der Entstehung des Wiener Positivismus in eine These umgewandelt, derzufolge diese und gewisse damit verbundene Eigenschaften, die nicht nur von den Brentanisten und den Wiener Positivisten, sondern auch von so unterschiedlichen Denkern wie Mach und Wittgenstein geteilt wurden, eine besondere Linie regionaler oder nationaler Philosophie in Österreich ausmachen. Die zahlreichen Schriften Hallers zur Geschichte dieser 'Österreichischen Philosophie' haben diese These weiter präzisiert, und durch die Gründung der Forschungsstelle und des Dokumentationszentrums für österreichische Philosophie in Graz wurde ihr sogar seine institutionelle Form gegeben. Seine Formulierungen wurdendann vor allem durch einige englische Philosophen weiter untermauert, die schließlich durch die sich daraus ergebende Verringerung des Stellenwerts der deutschen Philosophie nichts zu verlieren hatten. Wenn man allerdings die erwähnte These akzeptiert, also wenn man m.a.W. zugibt, daß es eine abgesonderte und in sich kohärente Tradition der 'österreichischen Philosophie' gibt, dann wird selbst der Wiener Kreis durch Brentano mit der Philosophie des katholischen Scholastizismus verbunden. Die Methode des offenen, demokratischen, gemeinsamen Philosophierens, des Arguments und Gegenarguments, erscheint somit als etwas, das nicht nur von Brentano und den Scholastikern des Mittelalters, sondern auch vonSchlick mit seinenDonnerstagabendveranstaltungenund vonWittgenstein in seiner Zelle in Cambridge gepflegt wurde.

\section{Probleme der Neurath-Haller-These}

Eine prägnante Formulierung dieser Neurath-Haller-These ${ }^{11}$ findet sich in Hallers Aufsatz "Wittgenstein und die 'Wiener Schule"” aus dem Jahre 1975:

Ich möchte ... zwei Thesen verteidigen: erstens, daß es eine eigenständige Entwicklung der Österreichischen Philosophie gegenüber demübrigen deutschsprachigen Raum seit hundert Jahren gegeben hat und zweitens, daß diese Entwicklung zu einem genetischen Erklärungsmodell führt, das

${ }^{11}$ Vgl. allerdings die folgenden Passagen eines Essays von Max Scheler, der 1915/16 veröffentlicht wurde:

Vor dem Krieg waren die Berührungen des höheren Geisteslebens überhaupt zwischen [Deutschland und Österreich] auffällig gering. In Philosophie und Nationalökonomie zum Beispiel fehlten sie fast völlig; selbst in den Fachkreisen waren die Methoden dieser und anderer Geisteswissenschaften sehr verschiedene und die Berührung nur äußerlich. Man denke nur an die Wirksamkeit Kants, die [in Deutschland] übermäßig, in Österreich gegenüber Herbart, Franz Brentano, dem englisch-französischen Positivismus ganz geringfügig war. ... Zwischen der österreichischen Philosophie, besonders der Brentanoschen Schule, und der deutschen herrscht bereits [im Jahre 1915] eine engere Wechselwirkung. Die unter Husserls Führung in Deutschland aufkeimende Phänomenologie hat ihre Keime in den Lehren Franz Brentanos und Bolzanos und ist durch diese Forscher hindurch auch mit gewissen Grundideen der Scholastik eng verknüpft. (Scheler 1915/16, S. 463)

Man sollte also vielleicht besser, wie Kevin Mulligan (1997, 2000) vorgeschlagen hat, von einer 'Scheler-Neurath-HallerThese' sprechen. 
eine immanente Homogenität der Österreichischen Philosophie bis hin zum Wiener Kreis und seinen Nachfahren zu behaupten gestattet. (1975, S. 92)

Die These hat sicherlich eine gewisse Plausibilität. Sie ist aber auch nicht ohne Probleme. Um nur ein Beispiel zu nennen: Obwohl es wahr ist, daß beiden Zusammenkünften des Wiener Kreises auch Werke Brentanos, Meinongs und Husserls behandelt wurden, wurden die entsprechenden Schriften zumindest imFall Brentanos und Husserls primär deswegen ausgewählt, weil Schlick sie mit besonderer Verachtung betrachtete.

Die These wurde auch von Friedrich Stadler angegriffen, der nicht bereit ist, die 'zwei Lager' der katholischen-reaktionären-bauernständischen und der progressiven-sozialistischen-positivistischen Kräfte in dieser Weise zusammenfließen zu sehen, wie dies die These zur Folge hat. Stadler hat deswegen darauf hingewiesen, daß im Gegensatz zum Bild des typischen österreichischen Philosophen, wie es Neurath und Haller gezeichnet haben, zumindest im offiziellenLeben der Wiener Universität inder Zeit zwischen 1918 und 1938 der Einfluß der logischen Positivisten oder der wissenschaftlichen Philosophie im allgemeinen verhältnismäßig gering war. Stadler hat weiter darauf hingewiesen, daß damals (wie bis in die jüngste Vergangenheit) sowohl in Vorlesungen als auch in Dissertationen die Geschichte der Philosophie einer etwas altmodischenSorte überwog, die von Kant, Schopenhauer, Spinoza, Platon, Nietzsche u. dgl. handelte. ${ }^{12}$ Vor dem Hintergrund dieser damals dominanten Universitätsphilosophie erscheint der Kreis um Schlick eigentlich als ein Haufen von Dilettanten, Kaffeehauswissenschaftlern, Mathematikern, darunter sogar Erfindern von "Kunstsprachen" - allesamt Leute, die als Philosophen erst später und zunächst nur außerhalb der Grenzen Österreichs ernst genommen wurden.

Eine etwas andere Art der kritischenBehandlung der Neurath-Haller-These hat mit der Tatsache zu tun, daß schon die Unterstellung, es gebe eine besondere Linie der 'österreichischen Philosophie', als Beleidigung der guten Bürger Österreichs empfunden werden müßte, da sie der These gleichkommt, daß die Philosophie in Österreich etwas ist, das außerhalb der großen Tradition der deutschsprachigen Philosophie liegt. Der gebildete Österreicher hält doch daran fest, daß die geistigen Traditionen seines Mutterlandes zur Linie Kants, Goethes, Fichtes, Lessings und Schillers gehören, daß auch er auf sein Bürgertum im berüchtigten Land der Dichter und Denker ein Anrecht hat. Von einem jungen österreichischen Philosophen zu verlangen, daß er aus Gründendes Nationalstolzes oder der Loyalität seine Energien in heimatliche Nebenströmungen investiert, wie es die von Otto Neurath oder Otto Weininger sind, implizierte doch eine nicht weniger radikale Einschränkung seiner Interessen, als wenn man jungen Literaturstudenten in Kanada oder Wales das Lesen Chaucers oder Shakespeares oder Miltons verbieten wollte.

\footnotetext{
${ }^{12}$ Stadler 1979, S. 43. Vgl. auch Menger 1994, S. 17. Es war in Reaktion gegen diese Vorherrschaft der 'kantischen' oder ähnlicher metaphysischer Strömungen, daß Schlick 1928 den Verein ‘Ernst Mach’ gründete.
} 


\section{Sprache und Stil}

Die auch heutzutage noch weit verbreitete spöttische Abweisung der Idee einer ‘österreichischenPhilosophie’ läßt sich sehr deutlich im Fall Edmund Husserls - des großen deutschsprachigen jüdisch-protestantischen Philosophen aus dem habsburgischen Mähren -, veranschaulichen. Die kürzlich veröffentlichte Edition der Briefe Husserls zeigt einen Denker, der sich selbst gerade als legitimen Erben der deutschen Kultur von Lessing, Herder, Schiller und Goethe betrachtete, und dem es selbstverständlich war, daß es 'die historische Mission des deutschen Volkes [ist], allen anderen Völkern in der Philosophie voranzuleuchten.' (Husserl 1994, Band V, S. 172) Sowenig wie sein Lehrer Brentano hat Husserl die Philosophie im Licht einer angeblichen Unterscheidung zwischen ‘österreichischen' und 'deutschen’ Traditionen begriffen, und wo er beispielsweise von 'meinem alten Österreich' spricht (Band IV, S. 179, Band III, S. 500), tut er dies ausschließlichim geographischen Sinn. Wie Meinong und Frege ist Husserl doch spätestens ab 1910 stolzer 'National-Deutscher' (in Gegensatz zu Meinong und Frege ist er allerdings nicht Antisemit). Wie fast alle Akademiker inDeutschland war er dementsprechend zu Beginn des Ersten Weltkrieges vonder Begeisterung des deutschen Nationalismus mitgerissen, und am Ende des Krieges setzte er seine Hoffnungen auf 'die ersehnte Vereinigung Deutsch-Österreichs und Deutschlands' (Band IX, S. 57).

Wie sich aber ein jeder leicht überzeugen kann, der mit Husserls früheren logischen Schriften oder mit den Werken Bolzanos, Brentanos oder Machs vertraut ist, bestehen radikale Unterschiede des Stils und der Art des Schreibens zwischen diesen deutschsprachigen Philosophen, die gewöhnlich mit Österreich assoziiert werden, und jenen (wie Hegel oder Heidegger), die zur deutschen Philosophie im engeren Sinn gezählt werden. Auf eine kurze Formel gebracht: Die erstgenannten, nicht aber die letztgenannten, verwenden eine nüchterne, wissenschaftliche Sprache. Es gibt auch die schon erwähnten Unterschiede, was die Rolle der Wissenschaft und Logik im Gegensatz zu Politik oder Ideologie in den beiden Traditionen angeht. Diese Unterschiede erklären auch, warum zwar deutsche, nicht aber österreichische Philosophen in den Kanon der 'Continental Philosophy' in Nordamerika aufgenommen wurden. Es sind Unterschiede, die, wie wir noch sehen werden, tief und historisch verwurzelt sind, und sie erklären auch, warum Deutschland - trotz der Tatsache, daß es solche Giganten der mathematischen Logik wie Frege, Hilbert und Gentzen hervorgebracht hat-, erst sehr spät eine Gemeinschaft analytischer Philosophen auf heimatlichem Boden zustande gebracht hat, und warum nicht wenige der dafür Verantwortlichen (wie Wolfgang Stegmüller) aus Österreich stammten. Wie Haller schreibt:

Wie wir gesehen haben, und immer leicht überprüfen können, bestimmt die Geographie der Katheder auch auf weite Strecken hin die geographische Wirkungsgeschichte von Ideen. Während in Deutschland Husserls und später Heideggers Einfluß wächst und bis zu den sechziger Jahren dieses Jahrhunderts beherrschend bleibt, hat die übrige Brentano-Schule so wenig wie die Philosophie des Wiener Kreises in jenen Breiten Fuß fassen können; der Empirismus scheint eben nicht in jedem 'Klima' zu gedeihen. (1975, S.171) 


\section{Der Fall Deutschland}

Vielleicht sollten wir also unsere Anfangsfrage bezüglichder Gründe, warum die wissenschaftliche Philosophie so tiefe Wurzeln im (katholischen) Österreich geschlagen hat, anders formulieren und stattdessen fragen, warum eine derartige Philosophie im nördlichen (lutheranischen) Gefilde des deutschen Sprachraums fast nirgends zur Blüte gekommen ist. Auch hier können wir uns zunächst an Neurath wenden, der eine Erklärung dieses Versagens bietet, die auf die religiösen Unterschiede hinweist:

Gerade Katholiken mit ihrer kompakten Dogmatik, die am Anfang ihrer Reflexionen steht, können zuweilen besonders unbeschwert von metaphysischen Einzelheiten sich logisch-systematischer Analyse widmen. ... [W]er im Bereich des Katholizismus überhaupt an einem Dogma zu zweifeln beginnt, befreit sich besonders leicht von der Gesamtheit der Dogmen und verfügt dann über ein sehr wirksames logisches Instrument als Restbestand! Anders im Bereich des Luthertums, wo die starre Dogmatik aufgelockert, die Kirchenmacht geschwächt wurde. Dort hat man, vielfach ein Bekenntnis zu einer klaren Dogmatik vermeidend, halbmetaphysische, viertelmetaphysische Wendungen als Restbestand unvollkommen verdrängter Theologie ... So mag es verständlich sein, daß gerade in Gebieten, in denen das Luthertum der katholischen Kirche die schwersten Schläge versetzte, die Sprachanalyse der Einheitswissenschaft sich amschwächstendurchsetzte, trotzder hochentwickelten Technik und der dazu gehörigen Wissenschaften. (Neurath 1933, S. 597)

Deshalb, behauptet Neurath in seiner charakteristisch voreiligen Art, gelingt 'die Auflehnung gegen metaphysische Tradition ... außerhalb des lutheranischen Bereiches in katholischen Gebieten ebenso in calvinistischen Gebieten', und mit Stolz bemerkt er, daß es in den genannten Gebieten 'keine Alleinherrschaft der Metaphysik [gebe], wie sie Rickert, Heidegger und andere ausüben’ (aaO.).

Leider war allerdings der junge Heidegger, Sohn des Meßners von Meßkirch, selbst von der katholischen Metaphysik geprägt. Schlick dagegen kam aus lutheranischem Hause. Und wie wir schon bemerkt haben, gibt es viele katholische, in anderer Hinsicht mit Österreich vergleichbare Länder, in denen logischer Empirismus und analytische Philosophie keine tiefen Wurzeln geschlagen haben, genauso wie es lutheranische Länder gibt - Finnland ist hier das auffallendste Beispiel-, wie auch natürlich (übrigens von Neurath gar nicht erwähnte) Länder anglikanischenBekenntnisses, die sich als wahrhafte Hochburgender analytischen Tradition ausgezeichnet haben.

Man muß also anderswo suchen, wobei sich herausstellt, daß Eigentümlichkeiten nicht der religiösen, sondern der politischen Geschichte der Deutschen für unser Problem von besonderer Bedeutung sind. Philosophie und Philosophen haben ja eine zentrale Rolle in der Gründung des deutschen Staates gespielt,

in einer Art, zu der sich kein Gegenstück in der Geschichte Englands oder Österreichs finden läßt (wohl aber in der des ehemaligen Sowjetrußland). Genau wie England seine Nationaltheater, hat Deutschland seine 
Nationalphilosophie. ${ }^{13}$ Kant, Fichte und Hegel sind wie Goethe und Schiller Volksheiligtiumer, und es ist jedes Deutschen Pflicht, ihnen ein ehrendes Gedenken zu bewahren, weil man glaubt, daß sie eine wesentliche Rolle inder Bildung des Bewußtseins der nationalenEinheit des deutschen Staates gespielt haben. Ihre Werke spielen im Geschichtsbewußtsein der Deutschen eine Rolle, die der der Werke Homers für die Griechen oder Shakespeares für die Engländer gleichkommt.

Man könnte nun glauben, daß die politisch-historische Resonanz und die mit ihr verbundenenEigenschaft der Kommentarbedürftigkeit verbundenen stilistischen Merkmale $^{14}$ der zentralen Texte der klassischen deutschen Philosophie eigentlich nebensächlich sind, daß sie mit dem Wesen dieser Philosophie nichts zu tun haben. Dagegen muß man allerdings bedenken, daß deutsche Philosophiestudenten in den letzten hundert Jahren in einer streng textorientierten philosophischen Kultur geschult wurden, deren wichtigste Texte sich durch eine markante, manchmal fast biblische Dichte und Tiefe und Undurchdringlichkeit, ja manchmal sogar durch poetische Qualitäten ('das seiende Sein, das nichtende Nichts') auszeichnen. Sie wachsen zudem in einer Kultur auf, die der 'Authentizität' einen äußerst hohen Wert zuschreibt: Sind doch Werke des Philosophen in einer solchen Kultur, wie die Werke des Dichters, Ausdruck des nationalen Geistes. (Lange vor Heidegger und in ungefähr demselben Ton hatte schon Herder auf dieser Tatsache insistiert.) Die Werke eines Meisters kommenschließlich direkt aus dem Herzen, sind Veräußerlichungen seiner Autorenseele. Man vergleiche in diesem Zusammenhang die quasi durch ein Verfahren automatisierten Schreibens produzierten Werke Derridas. Sie zeigen übrigens, wie sich die französische Philosophie (oder vielmehr: die Teile der französischen Philosophie, die als 'Continental Philosophy' etikettiert wurden), oft zur bloßen Karikatur ihrer großen deutschen Vorbilder degradiert. Teamarbeit, die Pflege einer gegenseitigen Kritik und der Beharrlichkeit des Argumentierens und Gegenargumentierens, überhaupt die Suche nach Wahrheit, sind vor diesem Hintergrund unvorstellbar. ${ }^{15}$

\footnotetext{
${ }^{13}$ Vgl. dazu auch Hook 1930.

${ }^{14}$ Diese stilistische Merkmale sind dann ihrerseits mit der Eigenschaft der Kommentarbedürftigkeit verbunden. Dazu Smith 1991a, 1992, 1994a, 1994b, Libera 1999.
} 
In der breiteren Welt ist es allerdings nicht die deutsche Philosophie mit ihren spezifischenhistorischen und politischen Assoziationen, sondern vielmehr die empirische oder besser: wissenschaftlich orientierte Philosophie, die seit einiger Zeit - ob zum Guten oder Schlechten - die Hauptströmung der gegenwärtigen Philosophie bildet. Letztere ist eine Philosophie, die die Werte der Logik, des Argumentierens, der technischen Kompetenz, der Internationalisierung und der Spezialisierung ${ }^{16}$ weit mehr schätzt, als die der literarischen, ideologischen und historischen Anknüpfungen, die in gewissen philosophischen Kreisen in Deutschland und Frankreich noch hoch im Kurs stehen.

Man könnte dementsprechend sagen, daß die prominentesten österreichischen Philosophen von Anfang an Prosa (das klare Wort) gesprochen haben, ohne es zu wissen. Oder, wenn wir denselben Punkt von einer anderen Seite aus betrachten: Es scheint, daß Hallers institutionelle Erklärung der Entstehung regionaler oder nationaler Philosophienin Europa eigentlichnicht als Erklärung des Falls Österreich angesehen werden sollte, sondern vielmehr auf die deutsche (und französische) Philosophie anzuwenden ist. Genau dort haben die politischen und literarischen (und daher regionalen) Verwicklungen des Fachs Philosophie beträchtliche Folgen, was in den letzten Jahre vor allem im Vergleich mit den angelsächsischen Ländern immer auffälliger geworden ist.

Die Frage der Entstehung der wissenschaftlichen Philosophie in Österreich oder anderswo bedarf nach dem bisher gesagten eigentlichkeiner Antwort. Eine derartige Entwicklung, ob in Österreich oder Polen oder Skandinavien, ist genau wie in England oder Nordamerika einfach ein unvermeidbarer Teil des allgemeinen Prozesses der wissenschaftlichen Spezialisierung. Der Terminus 'Österreichische Philosophie’, wie geeignet er auch immer sein mag, die Philosophien des habsburgischen Wien, Graz und Lemberg, der Bolzanisten, Machianer und Brentanisten zuzammenzufassen, ist insofern irreführend, als er suggeriert, daß es eine entsprechende regionale, ethnische oder sektiererische Philosophie gibt oder gegeben hat. Im Gegenteil: Die österreichische Philosophie vor allem der Vorkriegszeit ist Weltphilosophie, sie ist Philosophie an sich, sie ist einfach der Teil der deutschsprachigen Philosophie der letzten hundert Jahre, der im höchsten Maße den international gültigen Standards der Klarheit, Strenge und des Professionalismus entspricht.

\section{Literatur}

Ayer, Alfred J. 1977 Part of My Life, London: Collins.

Brentano, Franz 1968 Die vier Phasen der Philosophie, Hamburg: Felix Meiner.

\footnotetext{
${ }^{15}$ Vgl. Puntel 1991, Smith 1991.

${ }^{16}$ Dahms mißversteht die Bedeutung dieses letztgenannten Faktors, wenn er sein Bedauern zum Ausdruck bringt, daß 'die Akademisierung' des Wiener Kreises, die die Emigration vor allem in die Vereinigten Staaten mit sich brachte, 'auch die Vernachlässigung von Fragen zur Folge [hatte], die sich mit den sozialen Umständen und Folgen der Wissenschaft befaßten, wie sie für Neurath, Zilsel und Frank selbstverständlich gewesen waren.' (1987, S. 106. )
} 
Dahms, Hans-Joachim 1987 'Die Emigration des Wiener Kreises”, in F. Stadler (Hrsg.), Vertriebene Vernunft, I, 66-123.

Dahms, Hans-Joachim(Hrsg.) 1985 Philosophie, Wissenschaft, Aufklärung. Beiträge zur Geschichte und Wirkung des Wiener Kreises, Berlin/New York: Walter de Gruyter.

Dvorak, Johann 1985 "Wissenschaftliche Weltauffassung, Volkshochschule und Arbeiterbildung imWiender Zwischenkriegszeit. Am Beispiel von Otto Neurath und Edgar Zilsel”, in Dahms (Hrsg.), 129-143.

Engel, Pascal 1994 “The Decline and Fall of French Nietzscheo-Structuralism”, in Barry Smith(ed.), 1994, 21-41.

Flores-Morelos, Felipe 1996 "De intencionalidades y representaciones: de Franz Brentano a Sigmund Freud", Acheronta, 3, http//www.acheronta.org.

Good, David F. 1984 The Economic Rise of the Habsburg Empire: 1750-1914, Berkeley: University of California Press.

Haller, Rudolf 1975 'Wittgenstein und die 'Wiener Schule”, in W. Strolz und O. Schatz (Hrsg.), Dauer im Wandel.Aspekte österreichischer Kulturentwicklung, Wien-Freiburg-Basel, 137-162, zitiertnachder Neuausgabe in Haller 1979, 163-187.

Haller, Rudolf 1979 Studien zur österreichischen Philosophie, Amsterdam: Rodopi.

Haller, Rudolf 1993 Neopositivismus. Eine historische Einführung in die Philosophie des Wiener Kreises, Darmstadt: Wissenschaftliche Buchgesellschaft.

Haller, Rudolf und Rutte, Heiner 1977 “Gespräch mit Heinrich Neider: Persönliche Erinnerungen an den Wiener Kreis", Conceptus, 1, 21-42.

Hayek, Friedrich August 1966 "Diskussionsbemerkung über Ernst Mach und das sozialwissenschaftliche Denken in Wien", in Symposium aus Anlass des 50. Todestages von Ernst Mach, Freiburg i. Br.: ErnstMach-Institut, 41-44.

Hemecker, Wilhelm W. 1991 Vor Freud. Philosophiegeschichtliche Voraussetzungen der Psychoanalyse, München/Hamden/Wien: Philosophia.

Hook, Sidney 1930 “A Personal Impression of Contemporary German Philosophy”, The Journal of Philosophy, 27, 141-160.

Husserl, Edmund 1994 Briefwechsel (Husserliana Dokumente III), herausgegeben durch Karl Schuhmann in Zusammenhang mit Elisabeth Schuhmann, Dordrecht/Boston/London: Kluwer.

Libera, Alain de (1999): L'Art des généralités. Théories de l'abstraction, Paris: Aubier.

Menger, Karl 1994 Reminiscences of the Vienna Circle and the Mathematical Colloquium, herausgegeben durch L. Golland, B. McGuinness und A. Sklar, Dordrecht/Boston/London: Kluwer.

Mezei, Balázs und Smith, Barry (1998): The Four Phases of Philosophy, Amsterdam/Atlanta: Rodopi. Mulligan, Kevin 1993 “Post-Continental Philosophy: Nosological Notes”, Stanford French Review, 17, 
$133-150$.

Mulligan, Kevin (1997): 'Sur l'Histoire de l'approche analytique de l'histoire de la philosophie: de

Bolzano et Brentano à Bennett et Barnes", J.-M. Vienne (Hrsg.), Philosophie analytique et

Histoire de la philosophie, Paris: Vrin, 61-103.

Mulligan, Kevn (2000): "La Place de la philosophie Autrichienne", in K Mulligan und J.-P. Cometti

(Hrsg.) La Philosophie autrichienne, Paris: Vrin

Neurath, Otto 1933 Einheitswissenschaft und Psychologie, zitiert nach der Neuausgabe in Neurath

1981, Band I, 587-610.

Neurath, Otto 1942 "International Planning for Freedom", The New Commonwealth Quarterly, April

1942, 281- 292 und Juli 1942, 23-28, zitiert nach der Neuausgabe in O. Neurath, Empiricism and

Sociology, M. Neurath und R. S. Cohen, Hrsg., Dordrecht/Boston: D. Reidel, 1973, 422-440.

Neurath, Otto 1981 Gesammelte philosophische und methodologische Schriften (Haller und Rutte,

Hrsg.), 2 Bände, Wien: Hölder-Pichler-Tempsky.

Neurath, Otto, Carnap, Rudolf und Hahn, Hahn 1929 Wissenschaftliche Weltauffassung: Der Wiener

Kreis, Wien: Wolf; zitiert nach der Neuausgabe in Neurath 1981, Band I, 299-336.

Nyíri, J. C. 1986 “The Austrian Element in the Philosophy of Science”, in Nyíri (Hrsg.), 141-146.

Nyíri, J. C. (Hrsg.) 1981 Austrian Philosophy. Studies and Texts, München: Philosophia.

Nyíri, J. C. (Hrsg.) 1986 From Bolzano to Wittgenstein: The Tradition of Austrian Philosophy,

Wien: Hölder-Pichler-Tempsky.

Puntel, L. B. 1991 "The History of Philosophy in Contemporary Philosophy: The View from Germany”,

Topoi, 10, 147-154.

Scheler, Max 1915/16 "Soziologische Neuorientierung und die Aufgabe der deutschen Katholiken nach dem Kriege", Hochland, 13/1, 385-406 und 682-700, 13/2, 188-214, zitiert nach der Neuausgabe in Max Scheler, Gesammelte Werke, Band IV, Bern und München: Francke, 1982, 373-472.

Schuhmann, Karl und Smith, Barry 1985 “Against Idealism: Johannes Daubert vs. Husserl’s Ideas I”, Review of Metaphysics, 39, 763-93.

Smith, Barry 1991 “German Philosophy: Language and Style”, Topoi, 10, 155-161.

Smith, Barry 1991a "Textual Deference", American Philosophical Quarterly, 28, 1-13.

Smith, Barry 1992 “Thesen zur Nichtïbersetzbarkeit der deutschen Philosophie”, in D. Papenfuss und O. Pöggeler, Hrsg., Zur philosophischen Aktualität Heideggers, vol. 3, Im Spiegel der Welt: Sprache, Übersetzung, Auseinandersetzung, Frankfurt: Klostermann, 125-147.

Smith, Barry 1994 Austrian Philosophy: The Legacy of Franz Brentano, La Salle und Chicago: Open Court.

Smith, Barry 1994a “Über die Grenzen der Übersetzbarkeit”, in Armin Paul Frank, Kurt-Jürgen Maass, Fritz Paul und Horst Turk, Hrsg., Übersetzen. Verstehen. Brücken bauen. 
Geisteswissenschaftliches und literarisches Übersetzen im internationalen Kulturaustausch (Göttinger Beiträge zur Internationalen Übersetzungsforschung, 8/1), Berlin/Bielefeld/München: Erich Schmidt Verlag, 295-301.

Smith, Barry 1994b "Philosophieren und Kommentieren: Überlegungen zu ihrem Verhältnis", in H. F. Fulda und Rolf-Peter Horstmann (Hrsg.), Vernunftbegriffe in der Moderne. Stuttgarter HegelKongress 1993, Stuttgart: Klett Cotta, 857-868.

Smith, Barry (Hrsg.) 1994 European Philosophy and the American Academy, La Salle und Chicago: Open Court.

Stadler, Friedrich 1979 "Aspekte des gesellschaftlichen Hintergrunds und Standorts des Wiener Kreises am Beispiel der Universität Wien”, in H. Berghel, Hrsg., Wittgenstein, the Vienna Circle, and Critical Rationalism, Wien: Hölder-Pichler-Tempsky, 41-59.

Stadler, Friedrich 1986 Vom Positivismus zur "Wissenschaftlichen Weltauffassung": Am Beispiel der Wirkungsgeschichte von Ernst Mach in Österreich von 1895 bis 1934, Wien und München: Löcker.

Stadler, Friedrich (ed.) 1987 Vertriebene Vernunft (Veröffentlichungen des Ludwig BoltzmannInstitutes fur Geschichte der Gesellschaftswissenschaften. Sonderband, vol. 2), Wien: Jugend und Volk.

Stadler, Friedrich 1995 Wiener Kreis im Kontext. Ursprung und Entwicklung des logischen Empirismus, Frankfurt a. M.: Suhrkamp. 